\title{
Gut microbiota response to antibiotics is \\ personalized and baseline microbiota-dependent
}

\author{
Armin Rashidi \\ Maryam Ebadi \\ Tauseef Ur Rehman \\ Heba Elhusseini \\ Harika Nalluria \\ Thomas Kaiser \\ Shernan G. Holtan \\ Alexander Khoruts \\ Daniel Weisdorf \\ Christopher Staley
}

\section{Video Byte}

Keywords: antibiotics, leukemia, acute myeloid leukemia, AML, microbiota, 16S rRNA, sequencing, gut microbes, fecal, stool, chemotherapy, high-throughput, amplicon, precision treatment, dysbiosis, regime shift, microbiota perturbation, personalized response, baseline microbiota, Microbiome

Posted Date: March 1st, 2022

DOI: https://doi.org/10.21203/rs.3.rs-1405900/v1

License: (c) (1) This work is licensed under a Creative Commons Attribution 4.0 International License. Read Full License 


\section{Abstract}

Antibiotic treatment affects the gut microbiomes of different individuals to varying degrees. Such personalized responses may arise from differences in pretreatment microbiomes among individuals, but this possibility hasn't been directly assessed in humans. To test this hypothesis, a recent study used highthroughput 16S rRNA sequencing to analyze 260 stool samples from 39 patients with acute myeloid leukemia. Each patient received multiple antibiotics during an approximately 4-week hospital stay for chemotherapy, and the samples were collected twice weekly. The results revealed that the microbe communities were more similar among samples from the same patient than among samples from different patients, suggesting that the communities maintained some continuity despite the heavy antibiotic treatment. Statistical analyses showed that the antibiotic-induced microbiome changes were dependent on the pretreatment microbiome compositions and identified 6 taxa that predicted the extent of the changes. Although studies on more samples are needed, the results emphasize the importance of certain microbes in determining personalized responses to antibiotics and suggest that precision treatments targeting the pretreatment microbiota may help prevent antibiotic-induced microbiome disruption. 